University of Wollongong

Research Online

Faculty of Engineering and Information

Faculty of Engineering and Information

Sciences - Papers: Part B

Sciences

2010

On using human movement invariants to generate target-driven anthropomorphic locomotion

Manish Sreenivasa

University of Wollongong, manishs@uow.edu.au

Philippe Souères

LAAS-CNRS Toulouse France

Jean-Paul Laumond

LAAS-CNRS Toulouse France

Follow this and additional works at: https://ro.uow.edu.au/eispapers1

Part of the Engineering Commons, and the Science and Technology Studies Commons

Research Online is the open access institutional repository for the University of Wollongong. For further information contact the UOW Library: research-pubs@uow.edu.au 


\title{
On using human movement invariants to generate target-driven anthropomorphic locomotion
}

\begin{abstract}
We present a method for generating anthropomorphic motion by studying 'invariants' in human movements and applying them as kinematic tasks. We recorded whole-body motion of 14 participants during a walking and grasping task and performed a detailed analysis in order to synthesize the stereotypy in human motion as rules. We propose an algorithm that produces the key parameters of motion taking into account the knowledge from human movement, and the limitations of the anthropomorph. We generalize our results such that we can create motion parameters for objects which were not in the original protocol. The algorithmic output is applied in a task based prioritized inverse kinematics solver to generate dynamically stable and realistic anthropomorphic motion. We illustrate our results on the humanoid HRP-2 by making it walk to and grasp objects at various positions.
\end{abstract}

\section{Keywords}

locomotion, anthropomorphic, target-driven, generate, invariants, movement, human

\section{Disciplines \\ Engineering | Science and Technology Studies}

\section{Publication Details}

Sreenivasa, M. N., Soueres, P. \& Laumond, J. (2010). On using human movement invariants to generate target-driven anthropomorphic locomotion. 2010 3rd IEEE RAS \& EMBS International Conference on Biomedical Robotics and Biomechatronics (pp. 722-727). United States: IEEE. 


\title{
On using human movement invariants to generate target-driven anthropomorphic locomotion
}

\author{
Manish N. Sreenivasa, Philippe Souères and Jean-Paul Laumond
}

\begin{abstract}
We present a method for generating anthropomorphic motion by studying 'invariants' in human movements and applying them as kinematic tasks. We recorded whole-body motion of 14 participants during a walking and grasping task and performed a detailed analysis in order to synthesize the stereotypy in human motion as rules. We propose an algorithm that produces the key parameters of motion taking into account the knowledge from human movement, and the limitations of the anthropomorph. We generalize our results such that we can create motion parameters for objects which were not in the original protocol. The algorithmic output is applied in a task based prioritized inverse kinematics solver to generate dynamically stable and realistic anthropomorphic motion. We illustrate our results on the humanoid HRP-2 by making it walk to and grasp objects at various positions.
\end{abstract}

\section{INTRODUCTION}

Research in human locomotion is a rich source of inspiration for generating realistic anthropomorphic motion in, for example, computer graphics and humanoid robotics. There are several ways to go about implementing 'bio-inspired' motion. For example in computer graphics, several studies have developed methods to morph and/or combine recorded human motions to animate virtual figures [1],[2],[3]. In humanoid robotics, recent research has explored the idea of utilizing movements and principles from humans [4],[5],[6]. The organization of human locomotion (stepping) can also be used to direct similar behavior in anthropomorphs [7],[8].

In this study, we further incorporate human movement principles, for example that of the head and shoulders [9], to control target-driven locomotion in anthropomorphs. In a previous study we presented a robotics analogy to one such principle (head control during locomotion in humans), by controlling a complex 30 DoF humanoid robot, by simply translating and rotating its head [10]. However, since we only considered single steps in this approach, the generated motion was rather different from how humans move.

\section{A. Contribution}

We extend the envelope of our earlier study by looking at the organization of human motion at a more detailed level. The analogy here is between the concept of 'movement invariants' in human neuroscience, and 'kinematic tasks' in robotics. Our goals are to,

- Conduct detailed analysis of human movement during a target oriented walking and grasping task and extract the

This work was supported by the French ANR project LOCANTHROPE and the FUI project ROMEO.

Manish N. Sreenivasa, Philippe Souères and Jean-Paul Laumond are with CNRS; LAAS; 7 avenue du colonel Roche, F-31077 Toulouse, France. Universitè de Toulouse; UPS, INSA, INP, ISAE; LAAS; F-31077 Toulouse, France; manu/soueres/jplelaas.fr).
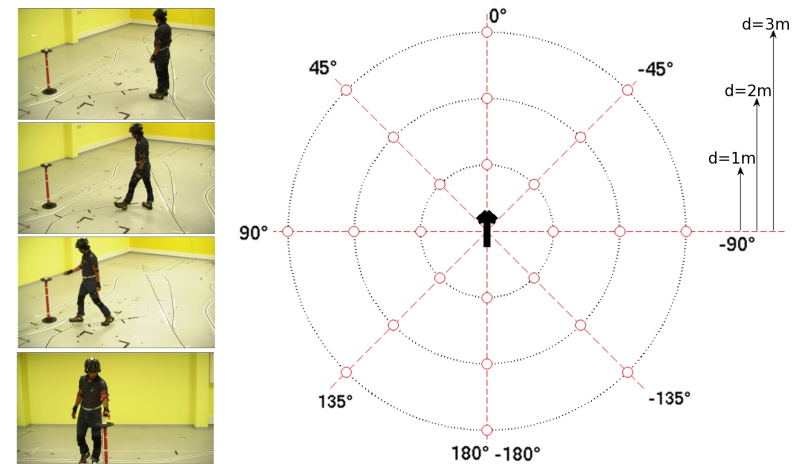

Fig. 1. Experimental Protocol: Snapshots of a participant while walking towards and grasping a cup placed on a pedestal. The pedestal was randomly placed around the participant at varying angles (dots) and radial distances (concentric dotted circles) relative to the participant's starting position and orientation (bold arrow).

'invariants' of this motion such that the overall behavior can be characterized into a set of rules.

- Develop an algorithm that implements these rules, generalizes them to targets outside the original protocol and takes into account the limitations of the target anthropomorph, to generate suitable motion parameters.

- Apply these motion parameters as kinematic tasks within the framework of a task based inverse kinematics solver to generate smooth and dynamic anthropomorphic motion which shares the 'essence' of the motion to that of humans.

In the following we describe the human movement experiments and their results, Section II. We then describe how these results are generalized to other anthropomorphs, Section III. In Section IV, we explain the methodology to generate anthropomorphic motion using a task-based prioritized inverse kinematics solver. Section V discusses our results and presents some perspectives for future research.

\section{HUMAN EXPERIMENTS}

We recorded motion of 14 participants $(8$ male, 6 females, mean demographics: 27 years, $63 \mathrm{~kg}, 1.7 \mathrm{~m}$ ) during a walking and grasping task. Participants volunteered to take part in the experiment and were naive with respect to its purpose. Written informed consent was sought and the experiment was conducted in accordance with the ethical standards laid down in the 1964 Declaration of Helsinki. In order to encode the key characteristics of our walking and grasping task, we took inspiration from human walking studies and chose the following parameters:

- Head, shoulder and pelvis orientation 

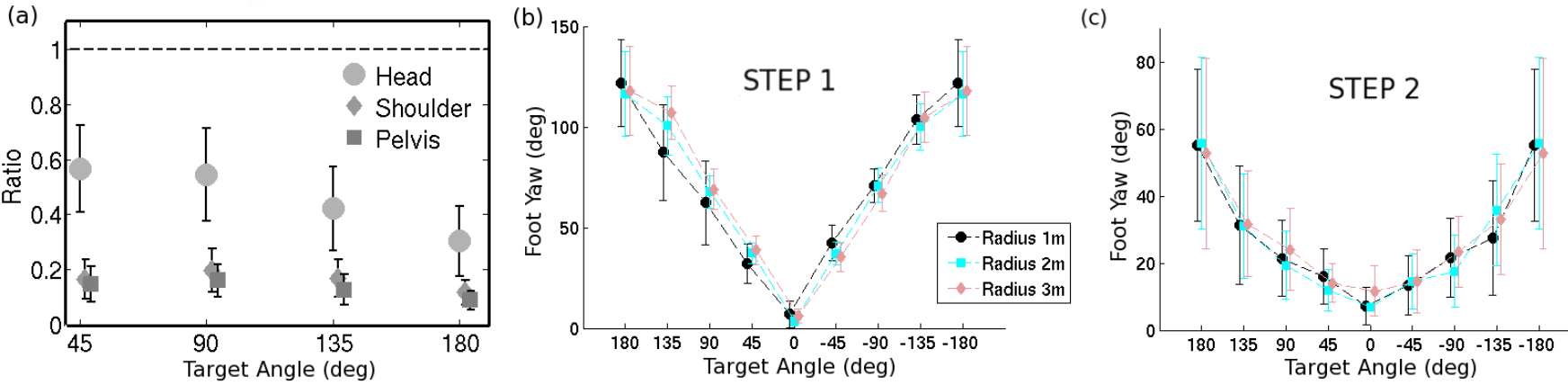

Fig. 2. (a) Ratio between the head, shoulder and pelvis yaw, and the absolute target angle, at the point of the start of stepping. (b) Yaw angle of first step with respect to the target angle. The three lines correspond to targets at 1, 2 and 3 m distance.(c) Yaw angle of second step (relative to yaw of first step) with respect to the target angle.

- Stepping positions and foot orientation

- Movement of the hand while grasping object

\section{A. Apparatus and Protocol}

The experiment was conducted in a $10 \times 6.5 \mathrm{~m}$ well lit empty walking space equipped with an optical tracking system (Motion Analysis Corporation, CA, USA). The tracking system consists of 10 infra-red cameras, which allow for an accuracy of position tracking of better than $1 \mathrm{~mm}$ in the entire walking area at a sampling rate of $100 \mathrm{~Hz}$. Participants wore a lightweight helmet $(\sim 200 \mathrm{~g})$ with 4 reflective tracking markers, and 20 additional markers were attached to track the positions of the shoulders, elbows, wrists, knees and legs.

Participants walked towards the pedestal $(0.9 m$ in height $)$ and picked up a plastic cup placed on it. The experiment was run in a single session of approximately 1 hour, including instructions and practice trials. The experiment followed an 8 Angle $\left(180^{\circ}, 135^{\circ}, 90^{\circ}, 45^{\circ}, 0^{\circ},-45^{\circ},-90^{\circ},-135^{\circ}\right) \times$ 3 Distance $(1,2,3 \mathrm{~m})$ design. Fig. 1 shows the layout of the experiment. The pedestal was placed at equal angular intervals with respect to the initial orientation of the participant. Each Angle and Distance pair was repeated 3 times and the order of trials was randomized. Each trial started with the participant standing in the middle of the walking space, fixating at a cross on the wall facing them. The experimenter then placed the pedestal at the position required for that trial. Participants were instructed to remain fixated on the cross until they heard a clearly audible beep. Upon hearing the sound, participants started walking towards the pedestal. They were asked to walk in a comfortable fashion and could use any hand to grasp the cup on the pedestal. There were no instructions as to which foot to use to initiate walking. The experimenter monitored all trials and stopped recording data once the cup was picked up. At the end of the trial the participant walked back to the starting point, fixated on the cross, and waited for the signal to start the next trial. All participants were given about 5 minutes of practice where they repeated a random set of trials.

\section{B. Data Analysis}

All analysis of motion capture data was done in Matlab R2007a (The Mathworks, USA). Raw marker data were low-pass filtered at $10 \mathrm{~Hz}$ to reduce electronic noise (3rd degree, zero phase shift Butterworth filter). Head position was calculated as the mid point of the 4 markers on the helmet. Head yaw was calculated for the local reference plane centered at this point. Using the relevant markers, midpoints and yaw of the shoulders, pelvis and feet were calculated in a similar manner.

Step detection: To robustly detect the start and end of stepping, we developed a step trigger based on a combination of foot Z-height, foot Z-velocity, foot yaw and foot yaw velocity. 'Step Start' was detected when foot Z-height or yaw angle increased beyond a certain threshold. By analyzing typical stepping behavior we chose a threshold of $2 \mathrm{~cm}$ for lift steps, and $5^{\circ}$ for yaw steps. Thresholds were set relative to the initial Z-height and yaw angle. To detect the end of the step, we first identified the instance when foot Z-velocity reduced below $0.05 \mathrm{~m} / \mathrm{s}$, and, the instance when the foot orientation velocity reduced below $50^{\circ} \%$ s. 'Step End' was set as the later of these two time instances. In order to avoid detecting the top of the foot step, where the foot Z-velocity and yaw velocity may also be zero, we made sure that the detected step end point occurs at a foot Z-height below the set threshold (initial Z-height $+2 \mathrm{~cm}$ ). The order of the stepping feet and the mean position and orientation of the foot, were calculated using these detected stepping events. Step yaw was calculated relative to the initial orientation of the foot at the beginning of the trial. Step size was the absolute distance between consecutive stepping positions.

\section{Results - Human Experiments}

Statistical analysis of the parameters was done with twoway ANOVAs using the statistical toolbox of Matlab R2007a. Except for turn direction and choice of first step, all parameters were collapsed across turn angles (i.e. $45^{\circ} \&-45^{\circ}$, $90^{\circ} \&-90^{\circ}$ etc) due to symmetry in results. Significance level was set at 0.05 . We report the results of the significant tests as $F(d f$,errord $f)$, where $F$ is a ratio of the variance between groups to the variance within groups, and, $d f$ and errordf are the between-groups and within-groups degrees of freedom for that test, respectively.

Turn Direction: Targets to the left of the participants resulted in left turns and vice-versa. For targets directly 
behind the participants $\left(180^{\circ}\right)$, we see an equal distribution of left and right turns. At an individual level we observed a highly systematic trend for all target angles except $180^{\circ}$. For this particular angle most participant's preferred to turn in one direction with no systematic trend across participants. In order to generalize turning behavior across participants, we chose to test the average data despite the variable individual behavior. For the average data, target angle had a significant effect on turn direction, $F(2,312)=272.6, p<0.05$, but distance did not have an effect, $p=0.18$. There was no interaction effect between angle and distance, $p>0.05$.

Head/Shoulder/Pelvis Yaw: Fig. 2-a shows the average ratio between the yaw of the head, shoulder and pelvis and the target angle. The results were collapsed across target angles since there was symmetric behavior for clockwise and counter-clockwise turns. The ratio for the head yaw reduced with target angle $\left(0.56\right.$ for $45^{\circ}$ to 0.3 for $\left.180^{\circ}\right)$, but when multiplied by the target angle this actually means an increase in absolute head yaw from $20^{\circ}$ to $60^{\circ}$, respectively. Similarly, shoulder and pelvis yaw increased for larger target angles, but their overall ratio was small (about 0.1). Target angle had a significant effect on the magnitude of yaw of the head, shoulder and pelvis before the start of stepping (all $p^{\prime} s<0.05$ ). Target distance had a significant effect on head yaw, $F(2,195)=3.09, p=0.047$, but not on shoulder and pelvis yaw $\left(p^{\prime} s>0.05\right)$. Multi-comparison tests revealed that head yaw for $d=1 m$ was the cause $\left(p^{\prime} s<0.05\right.$ for $d 1$ vs. $d 2$ and for $d 1$ vs. $d 3$ ). The difference between means of head yaw for $d 1 \& d 2$ was $6.7^{\circ}$, and $7.03^{\circ}$ for $d 1 \& d 3$.

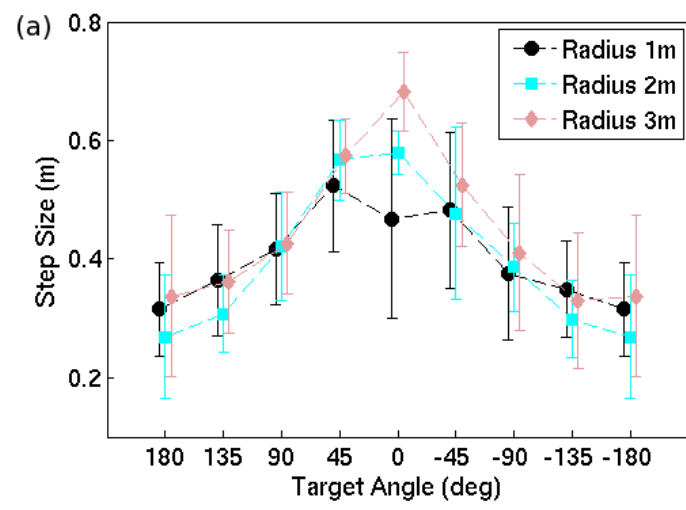

(b)

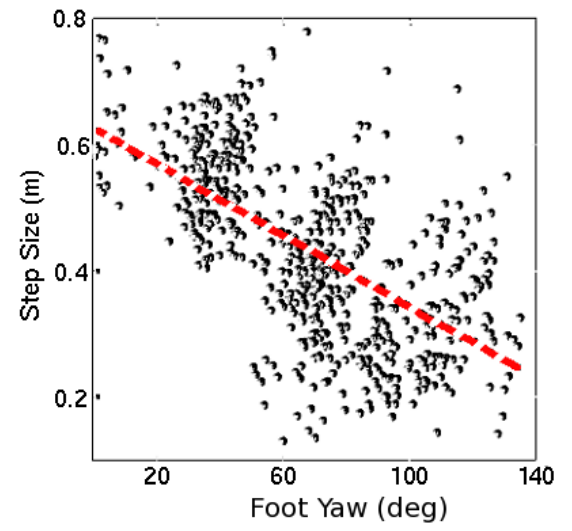

Fig. 3. (a) Variation of step size as a function of target angle and distance (b) Linear regression of step size vs. step yaw. Dots indicate the assimilated data points across all participants that were used in the regression.
Organization of Foot Steps: Target angle significantly affected foot used to start stepping , $F(7,312)=62.5, p<$ 0.05 , but this was not the case for target distance, $p=0.9$. There was no interaction effect between angle and distance. For targets in the forward half of the circle (i.e. $-90^{\circ}$ to $90^{\circ}$ ), first stepping foot was correlated with turn direction. For targets behind the participant there was an almost equal usage of left or right foot to start stepping, irrespective of whether the target was to the left $\left(135^{\circ}\right)$ or to the right $\left(-135^{\circ}\right)$ of the participant. Thus, for targets behind the participants, often the opposite foot to the turn direction was used to initiate stepping. To disambiguate we henceforth call normal stepping, where the foot used to step is the same as the turn direction as 'Step Turns', and the opposite case as 'Spin Turns'.

We observed that within the first two steps, the foot yaw reaches the target angle (Fig. 2-b \& c). For example, for a $135^{\circ}$ turn, the yaw of the first footstep is about $100^{\circ}$, and $32^{\circ}$ for the second. It is interesting to note that even for smaller targets, like $45^{\circ}$, the first foot step does not turn $45^{\circ}$ (although this is easily possible), but rather reaches the target angle on the second step. In spin turns, the foot rotates inwards and is limited by different bio-mechanical constraints than for step turns. Hence, we treat steps turns and spin turns separately (the data shown in Fig. 2-b \& c correspond to step turns). For both step and spin turns, magnitude of yaw in both the first and second steps changed significantly with target angle (all $p^{\prime} s<0.05$ ), but not with target radius (all $p^{\prime} s>0.05$ ). For spin turns, the magnitude of yaw for first and second steps were different than that for step turns (not shown), but the final foot yaw after two steps still reached the target angle.

Fig. 3-a shows the change in the size of the first step based on target angle and distance. Analysis showed that both target angle and target distance had a significant effect on step size, and there was also an interaction effect between the angle and distance (all $p^{\prime} s<0.05$ ). We note that for all target distances, step size decreased for larger target angles. Since step yaw increased for larger target angles, we present the step size as a function of step yaw (Fig. 3-b). Linear regression analysis confirmed that step size significantly decreased as step yaw increased $(t(648)=-19.33, p<0.001)$. Upon reaching the target angle, the subsequent steps (if required) were at close to maximum step size (about $0.7 \mathrm{~m}$ on average). We observed that often the final step was shorter, possible to bring the person within comfortable grasping range.

\section{Overall Behavior Description}

The results from the observation of human behavior during a walking and grasping task allows us to extract some behavioral 'invariants'. We can enumerate the general organization of movement in the following rules:

- Based on a target at a known angle relative to the person, a turn direction is chosen. If the target is directly behind, this choice is dependent on the individual, with most having a preferred turning direction.

- Before starting to step we rotate the head, shoulder and pelvis in the direction of the target. The magnitude of the head yaw depends on the angle of the target. The shoulder 
and pelvis also contribute to the overall yaw, but to a much lesser degree than the head.

- The choice of foot to start stepping depends on the target angle. For targets in the front of us, we usually start with the left or right leg, if the target is to the left or right, respectively. For targets behind us, we often first step inwards with the leg opposite to the turn direction.

- The first two foot steps are organized such that the orientation of the second step is close to the target angle required to reach. The organization of step turns is different from that for spin turns. Step size decreases with increasing step yaw.

- The hand grasp motion starts smoothly during the final hand swing and before the end of stepping.

\section{GENERALIZING HUMAN RESULTS TO OTHER} ANTHROPOMORPHS

In order to reproduce behavior similar to humans, the results detailed in the previous section need to be generalized to other anthropomorphic figures. Additionally, we would also like to generate the key characteristics for targets at all distances and angles.

Generalizing to all targets: Decisional parameters, like turn direction, were recorded as probabilities based on a given target angle. We assume the behavior at intermediate target angles to be continuous and approximate this by interpolating the recorded data points. Similarly, the other parameters were interpolated with respect to target angle. The only exception was step size, which was modeled with respect to step yaw and was calculated based on the slope of the regression line in Fig. 3-b.

Normalizing human results: In computer animation, studies have developed ways to transfer human motion to anthropomorphic figures by normalizing the motion [11]. We take inspiration from these studies to define the maximum limits of an average human. Limit for head yaw was set as $90^{\circ}$, and maximum shoulder and pelvis yaw were both set as $45^{\circ}$. Step size was normalized against a maximum value of $0.8 m$, similarly, maximum yaw for a step turn as $135^{\circ}$ and maximum yaw for a spin turn was $60^{\circ}$.

\section{A. Regenerating motion invariants}

Here, we use the generalized and normalized results from Sec. III to regenerate the key motion parameters for an anthropomorph. The inputs to the algorithm are the target angle, $\theta_{\text {targ }}$, and distance, $d_{\text {targ }}$, relative to the anthropomorph. Additionally, we have to take into account the limitations of the anthropomorph. For the parameters calculated we need to define the following limits:

$$
\left[\begin{array}{lllllll}
\theta_{m \_h d} & \theta_{m \_s h} & \theta_{m \_p l} & \theta_{m \_s t} & \theta_{m \_s p} & L_{m \_s t} & L_{g p}
\end{array}\right]
$$

where, $\theta_{m \_h d}, \theta_{m_{-} s h}$ and $\theta_{m_{-} p l}$, are the maximum permissible yaw of the head, shoulder and pelvis. $\theta_{m_{-} s t}$ and $\theta_{m_{-} s p}$ are the maximum possible outward and inward yaw of the feet, respectively. $L_{m_{-} s t}$ is the maximum step size possible, and $L_{g p}$ is the grasping range of the anthropomorph.

First, given a target angle and using the probability distributions described in the previous section, we predict the turning direction as turnDir $=p\left(\theta_{\text {targ }}\right) . p$ is the probability distribution of turning right as a function of angle. For, $\theta_{\text {targ }}=180^{\circ}$, we use the results of the average data across participants rather than setting a preferred turning direction, since, unlike humans, an anthropomorphic figure is symmetrical and the 'costs' of turning left or right in this case are equal. Second, we take into account the joint limits of the anthropomorph to calculate the head, shoulder and pelvis yaw before stepping:

$$
\left[\begin{array}{c}
\theta_{h d} \\
\theta_{s h} \\
\theta_{p l}
\end{array}\right]=\left[\begin{array}{c}
\alpha_{h d}\left(\theta_{\text {targ }}\right) \theta_{m_{-h d}} \\
\alpha_{s h}\left(\theta_{\text {targ }}\right) \theta_{m_{-s h}} \\
\alpha_{p l}\left(\theta_{\text {targ }}\right) \theta_{m_{-p l}}
\end{array}\right]
$$

where, $\alpha_{h d}\left(\theta_{\text {targ }}\right), \alpha_{s h}\left(\theta_{\text {targ }}\right)$ and $\alpha_{p l}\left(\theta_{\text {targ }}\right)$ are the normalized scale factors determined from the human experiments for a given $\theta_{\text {targ }}$.

Similar to turn direction, we choose the stepping foot using the results in Fig. 2. Steps were organized in three phases, the Orientation Phase (OP) which contributes to a major part of the orientation change towards the target, the Translation Phase (TP) that moves the body towards the target, and a final Grasping Phase (GP) that adjusts the distance from target in order to be at a comfortable grasping distance. For each phase we calculated the number of steps required based on the step yaw, spin yaw and step size limitations. Thus, for the orientation phase, number of steps $n_{O P}$ (rounded to the next integer) is:

$$
n_{O P}=\operatorname{int}\left(\theta_{\text {targ }} /\left(\theta_{m_{-s t}}+\theta_{m_{-} s p}\right)\right)+1
$$

where, $\operatorname{int}(.$.$) is the integer-part function. Step yaw and step$ size for the stepping foot were calculated relative to the support foot. Iteratively for each step $i=1, \ldots, n_{O P}$,

$$
\begin{aligned}
\theta_{i} & =\left\{\begin{array}{cc}
\alpha_{s t}\left(\theta_{\text {targ }}\right) \theta_{m_{-} s t} & \text { for step turns } \\
\alpha_{s p}\left(\theta_{\text {targ }}\right) \theta_{m \_s} & \text { for spin turns }
\end{array}\right. \\
L_{i} & =\alpha_{\text {st_size }}\left(\theta_{\text {targ }}\right) L_{m_{s} s t}
\end{aligned}
$$

where, $\theta_{i}$ is the yaw for the current step $i . \alpha_{s t}$ and $\alpha_{s p}$ are the normalized scale factors for step and spin turns calculated from human motion. $\alpha_{\text {st_size }}$ is the scale factor derived from the normalized value of the regression results shown in Fig. 3-b.

At the end of the orientation phase there is usually a small difference between the target angle and the cumulative yaw angle of the leading foot $\sum_{i=1}^{n_{o p}} \theta_{i}$ (due to the scale factor not allowing steps at maximum yaw). This difference, $\theta_{\text {targ }}-\sum_{i=1}^{n_{o p}} \theta_{i}$, is covered equally during the steps in the translation phase. The number of steps in the translation phase are calculated from the distance to target as,

$$
n_{T P}=\operatorname{int}\left(\left(d_{\text {targ }}-d_{O P}-L_{g p}\right) / L_{m \_s t}\right)+1
$$

where, $d_{O P}$ is the distance towards the target already covered in the orientation phase. Relative step size and yaw were calculated as in the previous phase. For the grasp phase (GP) the final step size is adjusted such that the final stepping foot is $0.1 \mathrm{~m}$ inside the grasping range (if it is not already within the grasping range). 

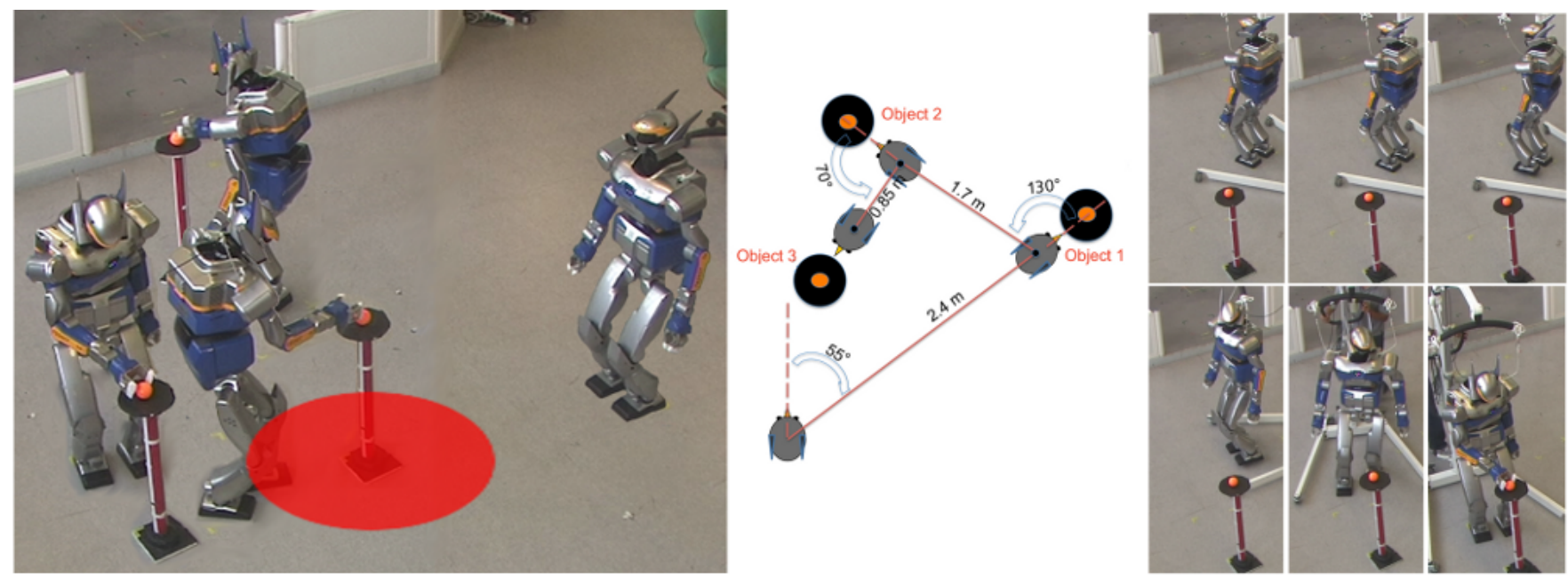

Fig. 4. Humanoid Experiments: Superimposed picture of the humanoid HRP-2 executing the motion generated using human movement invariants. The robot walked to and grasped three objects placed at pre-defined positions (picture on right). The translucent circle around the final object defines the comfortable grasping range of HRP-2, $0.5 \mathrm{~m}$ in this case, and the steps were organized such that one foot was inside this circle. Snapshots on the right show the humanoid as it turned and grasped object 2.

\section{HumANOID EXPERIMENTS}

In this study we illustrate the results of the algorithm on the humanoid robot HRP-2 (30 DoF, $56 \mathrm{~kg}, 1.5 \mathrm{~m}$ ). The parameters generated by our algorithm were applied as kinematic tasks and whole-body motion of the humanoid was generated using a task based prioritized inverse kinematics solver. Task based inverse kinematics solves the problem of redundancy in kinematic structures [12], like HRP-2, while allowing to simultaneously execute several tasks by giving each task a priority [13]. Dynamic stability of the humanoid was considered using the Zero Moment Point (ZMP) formulation [14],[15]. The overall motion of the humanoid was generated using an implementation of these principles in a package developed in a previous study [16]. A complete description of the dynamics computation and motion constraints is beyond the scope of this paper. Here, we list the tasks that were defined to derive humanoid motion.

\section{A. Whole-body motion generation}

Whole-body motion was generated from a prioritized stack of tasks, which was solved using the inverse kinematics formulation expressed above. For HRP-2, the limitations defined in (1) were: $\left[90^{\circ}, 45^{\circ}, 45^{\circ}, 45^{\circ}, 30^{\circ}, 0.23 \mathrm{~m}, 0.5 \mathrm{~m}\right]$. To implement the output of the algorithm in Section III-A we defined the following tasks/constraints:

1) Constraint to maintain waist Z-height ( $t=0$ to $T_{\text {end }}$ ) [2]

2) Constraint to maintain waist vertical ( $t=0$ to $T_{\text {end }}$ ) [2]

3) Constraint to maintain chest vertical $\left(t=0\right.$ to $\left.T_{\text {end }}\right)$ [2]

4) Head, Chest and Waist rotation task ( $t=0$ to $\left.T_{\text {gaze }}\right)$ [3]

5) Steps built from algorithm output ( $t=0$ to $T_{\text {stepEnd }}$ ) [1] with $n_{\text {steps }}=n_{O P}+n_{T P}+n_{G P}$

6) Head rotation task to fixate on the target ( $t=T_{\text {gaze }}$ to $\left.T_{\text {end }}\right)$ [3]

7) Grasping task ( $t=T_{\text {grasp }}$ to $\left.T_{\text {end }}\right)$ [2]

The tasks and constraints are presented in the chronological order of their starting time, and not in the order of their priority. The priority level for each task is indicated in the square brackets at the end. Priority level [1] is the highest and normally reserved for tasks dealing with stability. In this case the sequence of steps was given highest priority.

$T_{\text {gaze }}$ was the time allotted to rotate the head, chest and waist of HRP-2 to the values calculated by (2), and was set as 1 second. The grasp hand was chosen depending on the hand swing during walking. HRP-2's hands were controlled to swing in counter-phase to the stepping, and the hand which was in the down-swing phase at the time of final step was chosen for grasping, such that the overall motion of the grasping hand was smooth. By building successive steps to start one after the other, and solving the overall stack we can generate dynamic stepping motion while satisfying the other tasks/constraints in the stack. A more detailed explanation of the methodology of the motion generation can be found in [16], [17].

\section{B. Experimental Scenario}

We present a scenario where HRP-2 has to grasp 3 objects one after the other. The first object was located at $-55^{\circ}$ (i.e. clockwise) at a radial distance of $2.4 \mathrm{~m}$ from the robot's initial position and orientation. HRP-2 required 11 steps to grasp the object, with the first 3 being the orientation steps, followed by 7 translation steps and one grasping step. In order to illustrate movement where a big orientation change is required, the second object was placed at $130^{\circ}$ and $1.7 \mathrm{~m}$ relative to the final robot position of the previous stage. The robot completed the orientation phase in 5 steps, followed by 3 translation steps and one grasping step.The final object was located at $70^{\circ}$ and $0.85 \mathrm{~m}$ relative to the previous position and orientation. Since the object was close to the robot there were only 3 orientation steps and no translation steps or grasping step. Fig. 4 shows the position of the three objects along with the final position of the robot during the grasp. The red circle on the floor indicates the grasping range of HRP-2. The video of 
the humanoid and human experiments can be accessed at http://homepages.laas.fr/manu/videos/biorob2010.mp4.

In pilot trials we found that the final position of the humanoid's grasping hand was very close to that used in the algorithm (mean error about $5 \mathrm{~cm}$ ). However, grasping requires precise control of the hand and even small differences at the beginning of the motion (for example, due to slipping of the robot's feet on the ground) can cause errors that make the final grasp inaccurate. To solve this problem, we deliberately stop the grasping motion a little before the actual target. The final grasp is executed by using HRP-2's cameras which localize the object (an orange ball) in 3D and then automatically execute a precise grasping motion (see [18] for more details).

\section{CONCLUSIONS AND FUTURE WORK}

The approach presented in this study shows how anthropomorphic motion can be generated by using key information from the study of human movements. The idea of 'realism' of anthropomorphic motion is qualitative and hence difficult to prove mathematically. Several studies have developed parameters like the error between the joint angles (or positions) of the human and the figure being controlled [5],[4]. However, these parameters are often task-specific and cannot be compared across studies. Instead, we qualify our results by noting the following points. First, the humanoid HRP-2 walked to, and grasped, objects in the physical space having the same dimensions as the humans. Although the yaw of HRP-2's head, chest etc. were different from the humans, they were organized in the same way. The humanoid needed more steps to turn and walk than humans, but again the organization of these steps followed the same rules as in humans.

The results from our human walking experiments could also serve as a valuable knowledge base for guiding pure robotics based local optimization techniques like [19]. The drawback of using local optimization to generate motion for redundant anthropomorphic figures is that in certain cases it may reach the final goal, but in a manner completely different from that expected in humans, or fail altogether. By adding information from human movements these methods could be made more robust and the motion realistic.

In this study the walking was driven by a grasping task. It is important to note that the rules derived here as essentially independent of this grasping task. We could replace the final grasp with any other task that requires a focused visual attention. Related to the issue of visual attention, is the assumption we made that the head is driven by the gaze. Recording gaze during locomotion in humans is cumbersome, and most humanoids do not have vision independent of the head direction. For these reasons we limit our formulation to the orientation of the head, and base our assumption of synergistic eye-head movements on neuroscience results [20]. However, with the advent of robust eye-recording systems it would be interesting to complete our model by adding gaze information.

\section{ACKNOWLEDGEMENTS}

The authors would like to thank Oussama Kanoun, Anthony Mallet and Francisco Montecillo for taking part in helpful discussions and assisting with the experiments on HRP2, and Ilja Frissen for his help with the statistical analysis.

\section{REFERENCES}

[1] J. Pettre and J.-P. Laumond, "A motion capture-based control-space approach for walking mannequins," Comput. Animat. Virtual Worlds, vol. 17, no. 2, pp. 109-126, 2006.

[2] J. Lee, J. Chai, P. S. A. Reitsma, J. K. Hodgins, and N. S. Pollard, "Interactive control of avatars animated with human motion data," ACM Trans. Graph., vol. 21, no. 3, pp. 491-500, 2002.

[3] M. G. Choi, J. Lee, and S. Y. Shin, "Planning biped locomotion using motion capture data and probabilistic roadmaps," ACM Trans. Graph., vol. 22, no. 2, pp. 182-203, 2003.

[4] F.-J. Montecillo-Puente, M. Sreenivasa, and J.-P. Laumond, "On realtime whole-body human to humanoid motion transfer," in Informatics in Control, Automation and Robotics, International Conference on, 2010.

[5] B. Dariush, M. Gienger, B. Jian, C. Goerick, and K. Fujimura, "Whole body humanoid control from human motion descriptors," in Robotics and Automation, 2008. ICRA 2008. IEEE International Conference on, May 2008, pp. 2677-2684.

[6] K. Mombaur and M. Sreenivasa, "Hrp-2 plays the yoyo: From human to humanoid yoyo playing using optimal control," in Robotics and Automation, 2010. ICRA 2008. IEEE International Conference on, May 2010.

[7] R. Boulic, N. Magnenat-Thalmann, and D. Thalmann, "A global human walking model with real-time kinematic personification," Vis. Comput., vol. 6, no. 6, pp. 344-358, 1990.

[8] K. Harada, K. Miura, M. Morisawa, K. Kaneko, S. Nakaoka, F. Kanehiro, T. Tsuji, and S. Kajita, "Toward human-like walking pattern generator,' in IROS'09: Proceedings of the 2009 IEEE/RSJ international conference on Intelligent robots and systems. Piscataway, NJ, USA: IEEE Press, 2009, pp. 1071-1077.

[9] M. Sreenivasa, I. Frissen, J. Souman, and M. Ernst, "Walking along curved paths of different angles: the relationship between head and trunk turning." Exp Brain Res, vol. 191, no. 3, pp. 313-20, 2008.

[10] M.-N. Sreenivasa, P. Soueres, J.-P. Laumond, and A. Berthoz, "Steering a humanoid robot by its head," in Intelligent Robots and Systems, IROS 2009. IEEE/RSJ International Conference on, Oct. 2009.

[11] R. Boulic, D. Maupu, and D. Thalmann, "On scaling strategies for the full body interaction with virtual mannequins," Journal Interacting with Computers, Special Issue on Enactive Interfaces, vol. 21, no. 1-2, pp. 11-25, 2009.

[12] Y. Nakamura, Advanced Robotics: Redundancy and Optimization. Boston: Addison-Wesley Longman Publishing, 1991.

[13] B. Siciliano and J. Slotine, "A general framework for managing multiple tasks in highly redundant robotic systems," in Advanced Robotics, IEEE Internatioal Conference on, 1991, pp. 1211-1216.

[14] S. Kajita, F. Kanehiro, K. Kaneko, K. Fujiwara, K. Harada, K. Yokoi, and $\mathrm{H}$. Hirukawa, "Biped walking pattern generation by using preview control of zero-moment point," vol. 2, September 2003, pp. 16201626.

[15] M. Vukobratovic and J. Stepanenko, "On the stability of anthropomorphic systems," Mathematical Biosciences, vol. 15, pp. 1-37, 1972.

[16] E. Yoshida, O. Kanoun, C. Esteves, and J.-P. Laumond, "Taskdriven support polygon humanoids," in Humanoid Robots, IEEE-RAS International Conference on, 2006.

[17] O. Kanoun, "Task-driven motion control for humanoid robots," $\mathrm{Ph} . \mathrm{D}$. dissertation, LAAS-CNRS; Université de Toulouse, 2009.

[18] E. Yoshida, A. Mallet, F. Lamiraux, O. Kanoun, O. Stasse, M. Poirier, P. Dominey, J.-P. Laumond, and K. Yokoi, "Give me the purple ball he said to hrp-2 n.14," in Humanoid Robots, IEEE-RAS International Conference on, 2007.

[19] O. Kanoun, E. Yoshida, and J.-P. Laumond, "An optimization formulation for footsteps planning," in Humanoid Robots, IEEE-RAS International Conference on, 2009.

[20] T. Imai, S. Moore, T. Raphan, and B. Cohen, "Interaction of the body, head, and eyes during walking and turning." Exp Brain Res, vol. 136, no. 1, pp. 1-18, 2001. 